\title{
Comportamento agregativo em Subulina octona (Brugüière) (Mollusca, Subulinidae)
}

\author{
Sthefane D'ávila; Roberto Júnio P. Dias \& Elisabeth C. de A. Bessa \\ Departamento de Zoologia, Instituto de Ciências Biológicas, Universidade Federal de Juiz de Fora. Campus Universitário, \\ 36036-330 Juiz de Fora, Minas Gerais, Brasil. E-mail: sthefanedavila@hotmail.com
}

\begin{abstract}
Aggregative behavior in Subulina octona (Brugüière) (Mollusca, Subulinidae). The current study aimed to verify the existence of aggregative behavior in Subulina octona (Brugüière, 1789) and to obtain behavioral evidence of chemical mediation of this behavior. We used 150 adult animals allocated in 15 boxes with humus (10 animals per box). In each box we placed four identified plastic disks in an equidistant position. These disks worked as shelters for the animals. In experiment I, 10 animals were put in the middle of each box. After 24, 48, $96,120,144$, and 168 hours we observed the number of formed aggregations, as well as the position of the aggregates (in each shelter), the number of individual per aggregation and the number of isolated individuals. In order to test the hypothesis of conditioning of disks by chemicals compounds, the experiment II was carried out. All the individuals were transferred to other boxes with humus, three new disks and the disk containing the larger aggregate after the 168 hours of the experiment I (denominated conditioned disk). We verified, after 24 and 48 hours, the number of individuals below the conditioned disk. In experiment $I$, aggregations were observed in every used box and in every time intervals. In all boxes, there was a significant increase in the number of individuals per aggregation and a reduction in the number of isolated individuals until the end of the experiment (ANOVA, $\mathrm{p}<0.005$ ). In experiment II, after 24 hours, six from 15 boxes presented aggregations under the conditioned disk, and after 48 hours, seven boxes presented aggregations under conditioned disk. These were the aggregations with a larger quantity of individuals, which suggests the conditioning of the used disks by some factor, possibly a chemical compound.
\end{abstract}

KEY WORDS. Aggregation; chemical communication; land snail.

RESUMO. O objetivo deste trabalho foi verificar a existência de comportamento agregativo na espécie Subulina octona (Brugüière, 1789) e obter evidências da mediação química desse comportamento. Foram utilizados 150 animais adultos, distribuídos em 15 caixas com terra vegetal (10 animais por caixa). Em cada caixa foram colocados quatro discos plásticos identificados, em posição eqüidistante, os quais serviram como abrigos para os animais. No experimento I, foram colocados 10 animais no centro de cada caixa. Após 24, 48, 96, 120, 144 e 168 horas foram observados o número de agregados formados, a posição dos agregados (sob qual abrigo), o número de indivíduos por agregado e o número de indivíduos isolados. No experimento II, todos os indivíduos foram transferidos para outras caixas contendo terra vegetal, três novos discos e o disco que continha o maior agregado após as 168 horas do experimento I (denominado disco condicionado). Foi verificado, após 24 e 48 horas, o número de indivíduos sob o disco condicionado. Em todas as caixas utilizadas e em todos os intervalos de tempo observados no experimento I, houve a formação de agregados. Houve um aumento significativo do número de indivíduos por agregado e uma diminuição do número de indivíduos isolados com o passar do tempo (ANOVA, $\mathrm{p}<0,005$ ). No experimento II, após 24 horas, seis das 15 caixas apresentavam agregados sob o disco condicionado, e após 48 horas, sete caixas, sendo esses os agregados com maior número de indivíduos, o que sugere o condicionamento dos discos usados por algum fator, possivelmente um composto químico. PALAVRAS-CHAVE. Agregação; comunicação química; molusco terrestre.

O comportamento agregativo pode ser observado em gastrópodes terrestres e aquáticos (CHASE et al. 1980, CoOK 1992, Kleewein 1999, Ansell 2000, 2001, Fratini et al. 2001, Ковак 2001, Martell et al. 2002, Morton et al. 2002). Os fatores que favorecem a agregação incluem características do ambiente, tais como diferenças no microclima, heterogeneidade do habitat e distribuição em manchas de recursos; assim como fatores relacionados à reprodução e à sobrevivência dos moluscos, tais como a probabilidade de encontrar um parceiro sexual e o risco de dessecação (KleEwein 1999, CoOK 1992). Os moluscos podem se

Revista Brasileira de Zoologia 23 (2): 357-363, junho 2006 
agregar temporariamente para a reprodução, alimentação e repouso e durante períodos com maior risco de dessecação ou apresentar permanentemente uma distribuição agregada em uma área restrita (KLEEWEIn 1999, ANSELl 2000, 2001, Fratini et al. 2001, Ковак 2001, Martell et al. 2002, Morton et al. 2002). Os indivíduos de espécies com distribuição agregada apresentam pequenas áreas de vida, forte fidelidade a sítios com alimento, locais para repouso, oviposição e parceiros sexuais disponíveis (KLeEWEIN 1999).

A agregação pode ser estimulada por fatores químicos (Ansell 2000, 2001, Chevalier et al. 2000, Fratini et al. 2001, Morton et al. 2002) ou pelo contato físico entre indivíduos (Ковак 2001). Evidências a respeito da influência de compostos químicos sobre o comportamento agregativo de moluscos gastrópodes vêm sendo obtidas por meio de observações a campo e de experimentação em laboratório (CoOK 1992, Ansell 2000, Chevalier et al. 2000, Fratini et al. 2001, Vianey-Liaud \& Dussart 2002).

O objetivo deste trabalho foi verificar a existência de comportamento agregativo na espécie Subulina octona (Bruguière, 1789) (Subulinidae) e obter evidências da mediação química desse comportamento.

\section{MATERIAL E MÉTODOS}

Foram utilizados 150 animais adultos, obtidos do laboratório de Moluscos do Curso de Pós-graduação em Ciências Biológicas, Comportamento e Biologia Animal, Universidade Federal de Juiz de Fora, Minas Gerais, os quais forram distribuídos em 15 caixas ( $12 \mathrm{~cm}$ de diâmetro e $9 \mathrm{~cm}$ de profundidade) com terra vegetal esterilizada (10 animais por caixa). Em cada caixa foram colocados quatro discos plásticos (4 cm de diâmetro e $1 \mathrm{~cm}$ de altura) identificados (discos A, B, C e D), em posição eqüidistante, os quais serviram como abrigos para os animais. No experimento I, foram colocados 10 animais no centro de cada caixa. Após 24, 48, 96, 120, 144 e 168 horas foram observados o número de agregados formados, a posição dos agregados (sob qual disco), o número de indivíduos por agregado e o número de indivíduos isolados. Quando dois agregados foram formados em um mesmo intervalo de tempo e em uma mesma caixa, aquele com o maior número de indivíduos foi chamado de agregado 1, e o segundo, com número menor de indivíduos, agregado 2. No experimento II, os indivíduos foram transferidos para outras caixas, contendo terra vegetal, três novos discos e o disco que continha o maior agregado após as 168 horas do experimento I (denominado disco condicionado). Foi verificado, após 24 e 48 horas, o número de indivíduos sob o disco condicionado.

\section{RESULTADOS E DISCUSSÃO}

Em todas as caixas utilizadas e em todos os intervalos de tempo observados no experimento I houve a formação de agregados (Figs 1-2). Houve um aumento significativo do número de indivíduos por agregado ( $\mathrm{p}=0,000 ; \mathrm{F}=5,71)$ e uma diminui- ção do número de indivíduos isolados ( $\mathrm{p}=0,001 ; \mathrm{F}=4,75)$ com o passar do tempo (ANOVA, p < 0,005) (Tab. I). Nas caixas 1, 2, 6,13 e 15 , os discos sob os quais se formaram agregados com maior número de indivíduos nas primeiras 24 horas, continuaram abrigando agregados até o fim do experimento, existindo tendência ao aumento do número de indivíduos sob estes discos. O mesmo padrão foi observado nas caixas 3,8 e 9 , após 48 horas de experimento e nas caixas 4 e 5, após 96 e 108 horas de experimento, respectivamente. Nas caixas 10, 11 e 14, os maiores agregados foram observados sob diferentes discos ao longo do experimento, não sendo evidenciada preferência por um disco particular. Na caixa 7, o disco sob o qual se formou o maior agregado nas primeiras 24 horas continuou abrigando o maior número de indivíduos até as 108 horas de experimento. Após esse período, o maior agregado foi deslocado para outro disco (Tab. II). Esses resultados sugerem a existência de um estímulo à agregação nos locais com maior número de indivíduos.

A agregação pode representar para os moluscos terrestres uma estratégia para a conservação da água corporal. Os agregados usualmente envolvem muito contato entre os indivíduos, diminuindo a área de superfície corporal exposta e sujeita a evaporação (Dundee et al. 1975). Bohan et al. (2000) observaram que a agregação em Deroceras reticulatum (Müller) (Limacidae) e Arion intermedius (Normand,1852) (Arionidae) relaciona-se à umidade do solo.

CHASE et al. (1980) sugeriram que a agregação em Achatina fulica pode ter as funções de proteção contra a dessecação e contra predadores invertebrados.Do mesmo modo, em gastrópodes aquáticos, que ficam expostos durante a maré baixa, a agregação pode relacionar-se à proteção contra a dessecação (ANSEll 2001).

É provável que o comportamento agregativo favoreça o encontro de parceiros para o acasalamento. Hipoteticamente, os moluscos de diferentes espécies podem ter um padrão de distribuição permanentemente agregado, refletindo uma adaptação que ocorreu ao longo do tempo evolutivo ou se agregarem apenas durante o período reprodutivo, atraídos para seus coespecíficos por meio da liberação de feromônios. Além disso, é provável que o reconhecimento químico de indivíduos coespecíficos seja importante durante o processo de corte e cópula em espécies de moluscos terrestres. ADAMO \& CHASE (1988) e ReIse (1995) observaram uma fase inicial de reconhecimento, que precede a cópula em Helix aspersa Müller (Helicidae) e Derosceras rodnae Grossu \& Lupo, 1965 e D. praecox Wiktor,1966 (Agriolimacidae), respectivamente. Esse comportamento précópula envolvia, segundo ReIse (1995), uma "investigação mútua com os tentáculos". AdAMo \& CHASE (1988) também observaram que os moluscos se tocavam com os tentáculos e lábios.

No experimento II, após 24 horas, seis das 15 caixas apresentavam agregados sob o disco condicionado, e após 48 horas, sete caixas, sendo estes os agregados com maior número de indivíduos (Tab. III), o que sugere o condicionamento dos discos usados por algum fator químico. No presente estudo, não 

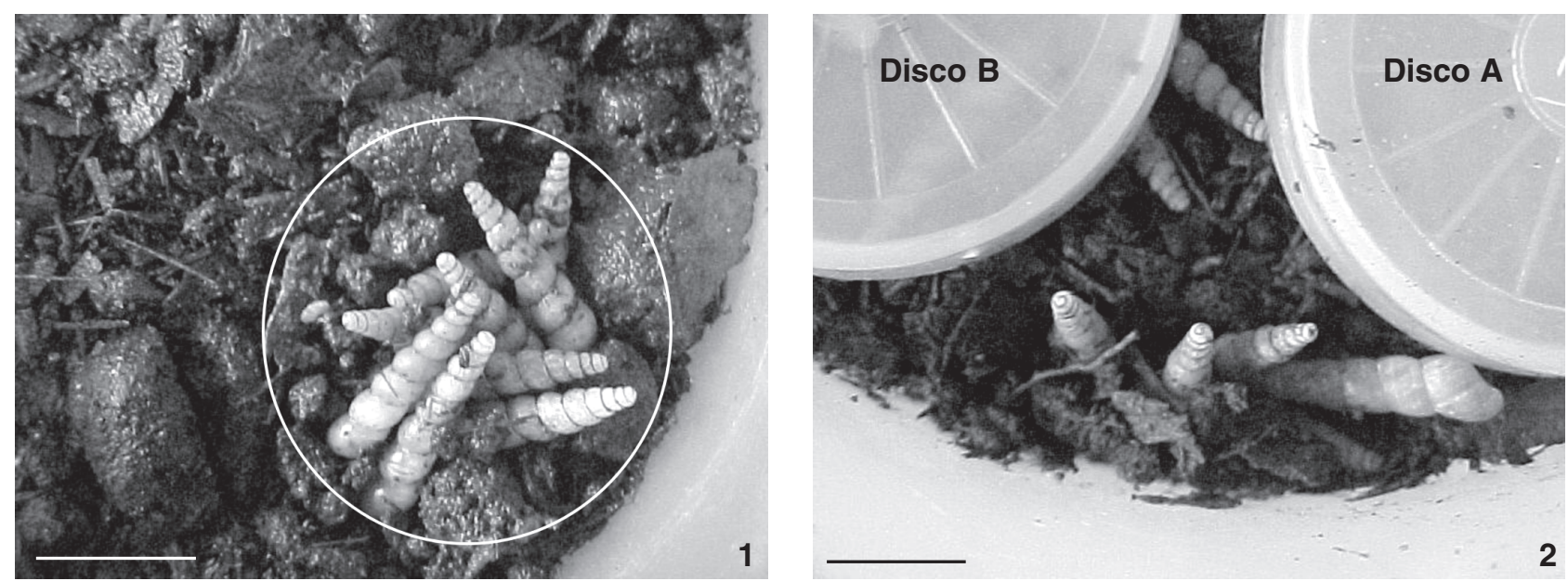

Figuras 1-2. Comportamento agregativo em Subulina octona: (1) agregado com nove indivíduos, formado sob o disco condicionado; (2) discos usados como abrigo, indivíduos agregados entre os discos A e B e sob o disco B. Barras $=1 \mathrm{~cm}$.

Tabela I. Número mínimo, máximo e médio de agregados, indivíduos por agregado e indivíduos isolados, da espécie Subulina octona, observados durante 168 horas.

\begin{tabular}{|c|c|c|c|c|c|c|c|c|c|c|c|c|}
\hline \multirow{3}{*}{ Tempo } & \multirow{2}{*}{\multicolumn{3}{|c|}{ Número de agregados }} & \multicolumn{6}{|c|}{ Número de indivíduos por agregado } & \multirow{2}{*}{\multicolumn{3}{|c|}{ Número de indivíduos isolados }} \\
\hline & & & & \multicolumn{3}{|c|}{ Agregado 1} & \multicolumn{3}{|c|}{ Agregado 2} & & & \\
\hline & Mín & Máx & Média & Mín & Máx & Média & Mín & Máx & Média & Mín & Máx & Média \\
\hline 24 horas & 1 & 2 & $1,66 \pm 0,48$ & 3 & 9 & $4,80 \pm 1,65$ & 0 & 3 & $2,00 \pm 1,46$ & 0 & 3 & $0,88 \pm 0,88$ \\
\hline 48 horas & 0 & 2 & $1,20 \pm 0,56$ & 0 & 9 & $5,20 \pm 2,36$ & 0 & 3 & $0,80 \pm 1,37$ & 0 & 4 & $1,73 \pm 1,09$ \\
\hline 96 horas & 1 & 2 & $1,30 \pm 0,48$ & 4 & 10 & $6,33 \pm 2,12$ & 0 & 5 & $1,40 \pm 2,09$ & 0 & 4 & $1,33 \pm 1,29$ \\
\hline 120 horas & 1 & 2 & $1,26 \pm 0,45$ & 5 & 10 & $7,13 \pm 1,95$ & 0 & 4 & $0,93 \pm 1,62$ & 0 & 3 & $1,06 \pm 0,96$ \\
\hline 144 horas & 1 & 2 & $1,26 \pm 0,45$ & 4 & 10 & $7,33 \pm 2,25$ & 0 & 4 & $0,93 \pm 1,62$ & 0 & 3 & $0,93 \pm 0,96$ \\
\hline 168 horas & 1 & 2 & $1,26 \pm 0,45$ & 5 & 10 & $8,13 \pm 2,13$ & 0 & 4 & $1,00 \pm 0,72$ & 0 & 2 & $0,66 \pm 0,72$ \\
\hline
\end{tabular}

foi possível determinar a natureza e origem de tal fator. O isolamento e estudo desse composto químico (ou compostos) em bioensaios futuros, permitirão elucidar se o estímulo à agregação em S. octona é provocado por um feromônio de agregação, como sugerido por DundeE et al. (1975) e CHASE et al. (1980), ou se os moluscos utilizam "pistas" químicas (não necessariamente feromônios) para detectarem a presença de coespecíficos e se agregarem.

Tais "pistas" químicas poderiam ser compostos liberados com o muco. O muco secretado pelos moluscos terrestres apresenta diferenças ao nível específico nos perfis de proteína e carboidrato. O muco depositado durante a locomoção é composto por lectinas, mucopolissacarídeos, glicoproteínas, proteínas, ácido urônico, ácido siálico, hexosaminas e uma variedade de outras moléculas, que podem constituir uma fonte de informações sobre a identidade e o estado fisiológico dos indivíduos (GAINEY 1976, SkIngsLey et al. 2000). Há evidências de que os moluscos são capazes de acessar, através do muco deixado por um coespecífico, o estado sexual do indivíduo e a direção da locomoção (TowSEnd 1974, CoOK 1992, KAROwe et al. 1993, SkINGSLEY et al. 2000). É provável que S. octona seja capaz de reconhecer a presença de um indivíduo da mesma espécie pela identificação das características do muco. Essa capacidade de reconhecer quimicamente coespecíficos poderia mediar a agregação.

A quimiorrecepção em gastrópodes pulmonados terrestres é bem desenvolvida. Toda a superfície externa do corpo desses moluscos é provida de células neurossensoriais, que são muito abundantes ao longo das margens do pé e na região anterior do corpo, incluindo os tentáculos e as pregas orais (HyMAN 1967, Chevalier et al. 2000). Entre os moluscos terrestres, a quimiorrecepção está associada à percepção do ambiente, à comunicação entre indivíduos de uma mesma espécie, ao comportamento de corte e cópula (COOK 1992, VIANEY-Liaud \& DusSART 2002), à escolha e encontro de alimentos (STEPHENSON 1979, Iglesias \& Castillejo 1999, Chevalier et al. 2000) e ao comportamento agregativo (DundeE et al. 1975).

Revista Brasileira de Zoologia 23 (2): 357-363, junho 2006 
Tabela II. Número de indivíduos da espécie Subulina octona, localizados sob os discos A, B, C e D, entre os discos (A e B, B e C, C e D, D e A) e no centro da caixa, durante 168 horas de observação.

\begin{tabular}{|c|c|c|c|c|c|c|c|c|c|c|c|c|c|c|c|c|c|c|c|}
\hline & $A$ & $A$ e $B$ & $B$ & $\mathrm{~B}$ e C & $C$ & Ce D & $\mathrm{D}$ & $\mathrm{DeA}$ & Centro & & $\mathrm{A}$ & $\overline{\mathrm{et}}$ & $\mathrm{B}$ & $\mathrm{B}$ e C & $C$ & Ce D & $\mathrm{D}$ & $\overline{D e A}$ & Centro \\
\hline Caixa 1 & & & & & & & & & & Caixa 8 (c & tin & Iaçã & & & & & & & \\
\hline $24 \mathrm{~h}$ & 3 & 1 & - & - & 6 & - & - & - & - & $108 \mathrm{~h}$ & - & - & 10 & - & - & - & - & - & - \\
\hline $48 \mathrm{~h}$ & - & - & 1 & - & 7 & - & 1 & - & - & $144 \mathrm{~h}$ & - & - & 10 & - & - & - & - & - & - \\
\hline $96 \mathrm{~h}$ & 1 & - & - & - & 9 & - & - & - & - & $168 \mathrm{~h}$ & - & - & 10 & - & - & - & - & - & - \\
\hline $108 \mathrm{~h}$ & - & - & - & - & 9 & - & 1 & - & - & Caixa 9 & & & & & & & & & \\
\hline $144 \mathrm{~h}$ & - & - & - & - & 9 & - & 1 & - & - & $24 \mathrm{~h}$ & - & - & - & 6 & 3 & - & - & - & - \\
\hline $168 \mathrm{~h}$ & - & 1 & - & - & 9 & - & - & - & - & $48 \mathrm{~h}$ & - & - & 9 & - & - & - & 1 & - & - \\
\hline Caixa 2 & & & & & & & & & & $96 \mathrm{~h}$ & 1 & - & 1 & - & 1 & - & 1 & - & 5 \\
\hline $24 \mathrm{~h}$ & - & - & - & - & - & 3 & 4 & 2 & - & $108 \mathrm{~h}$ & 1 & - & 5 & 5 & - & 1 & 1 & - & - \\
\hline $48 \mathrm{~h}$ & - & - & - & - & - & - & 5 & 2 & 1 & $144 \mathrm{~h}$ & 2 & - & 6 & - & - & - & 2 & - & - \\
\hline $96 \mathrm{~h}$ & - & - & - & - & - & - & 10 & - & - & $168 \mathrm{~h}$ & 3 & - & 7 & - & - & - & - & - & - \\
\hline $108 \mathrm{~h}$ & - & - & - & - & - & - & 10 & - & - & Caixa 10 & & & & & & & & & \\
\hline $144 \mathrm{~h}$ & - & - & - & - & - & - & 10 & - & - & $24 \mathrm{~h}$ & 3 & - & - & 4 & 2 & 1 & - & - & - \\
\hline $168 \mathrm{~h}$ & - & - & - & - & - & - & 10 & - & - & $48 \mathrm{~h}$ & 2 & - & - & 2 & 1 & 1 & 4 & - & - \\
\hline Caixa 3 & & & & & & & & & & $96 \mathrm{~h}$ & - & - & - & - & - & 5 & 5 & - & - \\
\hline $24 \mathrm{~h}$ & - & - & 1 & 3 & 2 & - & 3 & 1 & - & $108 \mathrm{~h}$ & 1 & - & - & - & 8 & - & 1 & - & - \\
\hline $48 \mathrm{~h}$ & - & - & 7 & - & 3 & - & - & - & - & $144 \mathrm{~h}$ & - & - & - & - & - & 5 & 5 & - & - \\
\hline $96 \mathrm{~h}$ & - & - & 9 & - & - & - & 1 & - & - & $168 \mathrm{~h}$ & - & - & - & - & 10 & - & - & - & - \\
\hline $108 \mathrm{~h}$ & - & - & 8 & - & 1 & - & 1 & - & - & Caixa 11 & & & & & & & & & \\
\hline $144 \mathrm{~h}$ & - & - & 8 & - & 2 & - & - & - & - & $24 \mathrm{~h}$ & 4 & 2 & - & - & 2 & - & 1 & 1 & - \\
\hline $168 \mathrm{~h}$ & - & - & 9 & - & - & - & 1 & - & - & $48 \mathrm{~h}$ & 2 & - & 2 & 2 & 1 & - & - & 1 & 2 \\
\hline Caixa 4 & & & & & & & & & & $96 \mathrm{~h}$ & 1 & 1 & 4 & - & - & - & 2 & 1 & 1 \\
\hline $24 \mathrm{~h}$ & 3 & - & 2 & 2 & 1 & - & 1 & 1 & - & $108 \mathrm{~h}$ & 3 & 1 & 6 & - & - & - & - & - & - \\
\hline $48 \mathrm{~h}$ & 1 & 1 & - & 2 & - & - & 2 & 1 & 3 & $144 \mathrm{~h}$ & - & - & - & - & - & - & - & 10 & - \\
\hline $96 \mathrm{~h}$ & 1 & 1 & - & - & 1 & - & 6 & - & - & $168 \mathrm{~h}$ & 1 & - & 9 & - & - & - & - & - & - \\
\hline $108 \mathrm{~h}$ & 2 & 1 & - & - & 1 & - & 7 & - & - & Caixa 12 & & & & & & & & & \\
\hline $144 \mathrm{~h}$ & - & - & - & - & 2 & 1 & 7 & - & - & $24 \mathrm{~h}$ & 7 & - & 3 & - & - & - & - & - & - \\
\hline $168 \mathrm{~h}$ & - & - & - & 1 & - & 1 & 8 & - & - & $48 \mathrm{~h}$ & 8 & - & 1 & - & - & - & - & - & 1 \\
\hline Caixa 5 & & & & & & & & & & $96 \mathrm{~h}$ & 6 & - & 2 & - & 2 & - & - & - & - \\
\hline $24 \mathrm{~h}$ & 2 & - & 1 & 2 & - & - & - & 1 & 4 & $108 \mathrm{~h}$ & - & - & 8 & - & - & - & 2 & - & - \\
\hline $48 \mathrm{~h}$ & - & 6 & - & - & - & 1 & 3 & - & - & $144 \mathrm{~h}$ & 3 & 1 & 5 & - & - & - & 1 & - & - \\
\hline $96 \mathrm{~h}$ & - & 1 & 1 & - & - & - & 4 & 2 & 1 & $168 \mathrm{~h}$ & - & - & 10 & - & - & - & - & - & - \\
\hline $108 \mathrm{~h}$ & 5 & 2 & - & - & 1 & 2 & - & - & - & Caixa 13 & & & & & & & & & \\
\hline $144 \mathrm{~h}$ & 6 & - & - & - & - & 1 & - & 2 & 1 & $24 \mathrm{~h}$ & 1 & - & 6 & - & 3 & - & - & - & - \\
\hline $168 \mathrm{~h}$ & 5 & - & - & - & - & - & 4 & 1 & - & $48 \mathrm{~h}$ & 1 & 2 & 3 & - & 3 & - & - & - & 1 \\
\hline Caixa 6 & & & & & & & & & & $96 \mathrm{~h}$ & 1 & - & 5 & - & 3 & - & - & - & - \\
\hline $24 \mathrm{~h}$ & 4 & - & 2 & 3 & 1 & - & - & - & - & $108 \mathrm{~h}$ & 1 & - & 5 & - & 3 & 1 & - & - & - \\
\hline $48 \mathrm{~h}$ & 6 & - & 2 & - & - & - & 1 & - & 1 & $144 \mathrm{~h}$ & 3 & - & 4 & - & 2 & 1 & - & - & - \\
\hline $96 \mathrm{~h}$ & 10 & - & - & - & - & - & - & - & - & $168 \mathrm{~h}$ & 2 & - & 5 & - & 2 & 1 & - & - & - \\
\hline $108 \mathrm{~h}$ & 10 & - & - & - & - & - & - & - & - & Caixa 14 & & & & & & & & & \\
\hline $144 \mathrm{~h}$ & 10 & - & - & - & - & - & - & - & - & $24 \mathrm{~h}$ & 4 & 2 & 2 & - & - & - & - & 2 & - \\
\hline $168 \mathrm{~h}$ & 10 & - & - & - & - & - & - & - & - & $48 \mathrm{~h}$ & 4 & - & 1 & 1 & - & - & 2 & 1 & 1 \\
\hline Caixa 7 & & & & & & & & & & $96 \mathrm{~h}$ & 5 & - & 5 & - & - & - & - & - & - \\
\hline $24 \mathrm{~h}$ & 9 & - & - & - & 1 & - & - & - & - & $108 \mathrm{~h}$ & 4 & - & 6 & - & - & - & - & - & - \\
\hline $48 \mathrm{~h}$ & 2 & - & - & 2 & 1 & 1 & 4 & - & - & $144 \mathrm{~h}$ & 5 & 1 & 4 & - & - & - & - & - & - \\
\hline $96 \mathrm{~h}$ & 5 & - & 4 & - & 1 & - & - & - & - & $168 \mathrm{~h}$ & 5 & - & 4 & - & - & - & 1 & - & - \\
\hline $108 \mathrm{~h}$ & 5 & - & 4 & - & 1 & - & - & - & - & Caixa 15 & & & & & & & & & \\
\hline $144 \mathrm{~h}$ & - & 1 & 9 & - & - & - & - & - & - & $24 \mathrm{~h}$ & 1 & 3 & - & 4 & - & 1 & - & 1 & - \\
\hline $168 \mathrm{~h}$ & - & - & 10 & - & - & - & - & - & - & $48 \mathrm{~h}$ & 3 & 3 & - & 1 & - & 2 & - & 1 & - \\
\hline Caixa 8 & & & & & & & & & & $96 \mathrm{~h}$ & 2 & 6 & 1 & - & 1 & - & - & - & - \\
\hline $24 \mathrm{~h}$ & - & 4 & 3 & 1 & - & - & - & 2 & - & $108 \mathrm{~h}$ & 1 & 6 & - & 1 & - & - & - & 1 & - \\
\hline $48 \mathrm{~h}$ & - & - & 10 & - & - & - & - & - & - & $144 \mathrm{~h}$ & 2 & 7 & - & - & - & - & 1 & - & - \\
\hline $96 \mathrm{~h}$ & - & - & 5 & 4 & - & - & 1 & - & - & $168 \mathrm{~h}$ & 4 & 5 & - & - & - & - & 1 & - & - \\
\hline
\end{tabular}

Revista Brasileira de Zoologia 23 (2): 357-363, junho 2006 
Tabela III. Número de indivíduos da espécie Subulina octona localizados sob o disco onde se formou o maior agregado no experimento I (disco condicionado), após 24 e 48 horas do início do experimento II.

\begin{tabular}{|c|c|c|c|c|c|c|c|c|c|}
\hline \multirow{2}{*}{$\begin{array}{l}\text { Tempo de } \\
\text { observação }\end{array}$} & \multicolumn{9}{|c|}{ Distribuição espacial dos moluscos } \\
\hline & Disco A & Entre disco A-B & Disco B & Entre disco B-C & Disco C & Entre disco C-D & Disco D & Entre disco D-A & Centro da caixa \\
\hline \multicolumn{10}{|l|}{24 horas } \\
\hline Caixa 1 & 1 & - & - & - & $9^{*}$ & - & - & - & - \\
\hline Caixa 2 & 2 & - & - & - & 3 & - & $5^{*}$ & - & - \\
\hline Caixa 3 & 2 & 1 & 3 & - & 1 & - & 3 & - & - \\
\hline Caixa 4 & 4 & 1 & 3 & - & - & 1 & 1 & - & - \\
\hline Caixa 5 & $5^{*}$ & - & 2 & - & - & 1 & 1 & 1 & - \\
\hline Caixa 6 & 1 & 1 & 3 & 1 & 2 & - & 1 & 1 & - \\
\hline Caixa 7 & 1 & 2 & - & - & 1 & - & 2 & - & 1 \\
\hline Caixa 8 & - & - & $4^{*}$ & - & 3 & - & 2 & - & 1 \\
\hline Caixa 9 & - & - & - & 3 & 2 & - & 4 & 1 & - \\
\hline Caixa 10 & 2 & - & 7 & - & 1 & - & - & - & - \\
\hline Caixa 11 & 2 & - & $7^{*}$ & - & - & - & 1 & - & - \\
\hline Caixa 12 & - & - & - & - & 8 & - & 2 & - & - \\
\hline Caixa 13 & 2 & - & $6^{*}$ & 2 & - & - & - & - & - \\
\hline Caixa 14 & - & 4 & - & 1 & - & - & 1 & 3 & 1 \\
\hline Caixa 15 & - & - & - & - & 10 & - & - & - & - \\
\hline \multicolumn{10}{|l|}{48 horas } \\
\hline Caixa 1 & 1 & - & - & - & $7^{*}$ & 1 & - & 1 & - \\
\hline Caixa 2 & - & - & - & - & - & - & $10^{*}$ & - & - \\
\hline Caixa 3 & 4 & - & 1 & - & - & 2 & 3 & - & - \\
\hline Caixa 4 & 4 & - & 4 & 1 & 1 & - & - & - & - \\
\hline Caixa 5 & $8^{*}$ & - & 1 & - & 1 & - & - & - & - \\
\hline Caixa 6 & $4^{*}$ & - & 2 & - & 4 & - & - & - & - \\
\hline Caixa 7 & 2 & - & 1 & - & 3 & - & 4 & - & - \\
\hline Caixa 8 & 3 & - & $4^{*}$ & - & 1 & - & - & - & 2 \\
\hline Caixa 9 & - & - & $3^{*}$ & 1 & 2 & - & 3 & - & - \\
\hline Caixa 10 & 2 & - & 5 & 1 & - & - & 1 & - & 1 \\
\hline Caixa 11 & 3 & - & $3^{*}$ & 2 & - & - & 2 & - & - \\
\hline Caixa 12 & - & - & - & 1 & 6 & - & 2 & 1 & - \\
\hline Caixa 13 & 3 & 3 & 2 & 2 & - & - & - & - & - \\
\hline Caixa 14 & - & 1 & - & 1 & - & - & 5 & 3 & - \\
\hline Caixa 15 & - & - & - & - & 10 & - & - & - & - \\
\hline
\end{tabular}

*Número de indivíduos dos agregados formados sob o disco condicionado.

Os moluscos terrestres são capazes de reconhecer compostos químicos voláteis e não-voláteis por dois mecanismos distintos: a percepção olfativa e gustativa, respectivamente (Stephenson 1979, Iglesias \& Castillejo 1999, Chevalier et al. 2000). STEPHENSON (1979) obteve evidências comportamentais do papel dos tentáculos anteriores e posteriores na detecção de compostos químicos voláteis e não-voláteis, assim como da função gustativa da probóscide, lábios e lobos laterais da boca de moluscos da espécie Deroceras reticulatum (Müll.). O autor ob- servou a presença de canais no prossoma, ao longo dos quais, muco com 90-95\% de água era direcionado para os órgãos sensoriais (probóscide e tentáculos), garantindo que tais órgãos fossem constantemente banhados por esse meio líquido. Com base nestas observações, propôs uma hipótese para explicar o mecanismo de detecção de compostos não-voláteis, segundo a qual, o muco presente nos órgãos sensoriais e a água presente na superfície das plantas, quando em contato, formariam uma ponte aquosa. A percepção de compostos químicos derivados 
das plantas, dissolvidos na água, ocorreria por meio do fluxo dessas substâncias através da ponte aquosa, até os órgãos olfativos. $\mathrm{O}$ autor sugeriu que este mecanismo provavelmente era utilizado pelos ancestrais aquáticos dos moluscos terrestres.

Assim como nos gastrópodes pulmonados terrestres, a percepção química é o principal sentido utilizado por gastrópodes prosobrânquios aquáticos para o encontro de alimento. Existem evidências comportamentais da capacidade desses moluscos perceberem compostos químicos dispersos na água, assim como do papel da estimulação química para a formação de agregados (Fratini et al. 2001, Morton et al. 2002). Entretanto, ainda não há uma clara resolução do mecanismo e estruturas sensoriais envolvidas na quimiopercepção por estes gastrópodes. A elucidação desse processo poderia mostrar se a especulação de STEPHENSON [sic], sobre a retenção do mecanismo ancestral de percepção de compostos não-voláteis pelos moluscos terrestres, corresponde à verdade. Fratini et al. (2001) relataram que Terebralia palustris (Linnaeus) (Potamidae), gastrópode prosobrânquio marinho, pode utilizar odores dispersos pelo ar para localizar alimento, durante a maré baixa. Tal observação pode indicar que os mecanismos sensoriais envolvidos na percepção química, e os comportamentos influenciados por essa percepção, talvez não sejam completamente distintos entre pulmonados terrestres e prosobrânquios aquáticos. A constatação de tal hipótese pode trazer importantes implicações para a compreensão da história evolutiva dos gastrópodes pulmonados e das pressões seletivas envolvidas na transição para o ambiente terrestre.

\section{CONSIDERAÇÕES FINAIS}

Os resultados desse estudo evidenciam o comportamento agregativo em $S$. octona e sugerem a mediação química desse comportamento. A agregação, aliada ao hermafroditismo parece ser uma estratégia para viabilizar a reprodução em animais com capacidade de deslocamento limitada, tais como os moluscos terrestres. O comportamento agregativo em S. octona pode ser o reflexo de um padrão de distribuição agregada da espécie, assim como uma estratégia para a resistência à dessecação e para o encontro de parceiros sexuais.

\section{REFERÊNCIAS BIBLIOGRÁFICAS}

Adamo, S.A. \& Chase, R. 1988. Courtship and copulation in the terrestrial snail Helix aspersa. Canadian Journal of Zoology, Toronto, 66: 1446-1453.

Ansell, A.D. 2000. Drilling and nondrilling predation in gastropod aggregations in the intertidal zone of a New Zeland harbour. Journal of Molluscan Studies, Londres, 66: 419422.

AnselL, A.D. 2001. Dynamics of aggregation of a gastropod predator/scavenger on a New Zeland harbour beach. Journal of Molluscan Studies, Londres, 67: 329-341.

Bohan, D.A.; D.M. Glen; C.W. Wiltshire \& L. Hughes. 2000.
Parametric intensity and the spatial arrangement of the terrestrial herbivores Deroceras reticulatum and Arion intermedius. Journal of Animal Ecology, Oxford, 69: 1031-1046.

Chase, R.; R.P. Croll \& L.L. Zeichner. 1980. Aggregation in snails, Achatina fulica. Behavioral and Neural Biology, New York, 30: 218-230.

Chevalier, L.; C. Desboquois; J. Papineau \& M. Charrier. 2000. Influence of the quinolizidine alkaloid content of Lupinus albus (Fabaceae) on the feeding choice of Helix aspersa (Gastropoda: Pulmonata). Journal of Molluscan Studies, Londres, 66: 61-68.

Cook, A. 1992. The function of trail following in the pulmonate slug Limax pseudoflavus. Animal Behaviour, Nedlands, 43: 813-821.

Dundee, D.S.; M. Tizzard \& M. Traub. 1975. Aggregative behaviour in veronicellid slugs. The Nautilus, Sanibel, 89 (3): 69-71.

Fratini, S.; S. CanNicci \& M. Vannini. 2001. Feeding clusters and olfactionin the mangroove snail Terebralia palustris (Linnaeus) (Potamidae: Gastropoda). Journal of Experimental Marine Biology and Ecology, Sydney, 261: 173-183.

Gainey, L.F. 1976. Locomotion in the Gastropoda: functional morphology of the foot in Neritina reclivata and Thais rustica. Malacologia, Ann Arbor, 15 (2): 411-431.

Hyman, L.H. 1967. The invertebrates: Mollusca I. New York, MC Graw-Hill, vol. 6, 792p.

Iglesias, J. \& J. Castillejo. 1999. Field observations on feeding of the land snail Helix aspersa Müller. Journal of Molluscan Studies, Londres, 65: 411-423.

Karowe, D.N.; A.T. Pearce \& W.R. Spaller. 1993. Chemical communication in freshwater snails: behavioral responses of Physa parkeri to mucous trails of $P$. parkeri (Gastropoda: Pulmonata) and Campeloma decisum (Gastropoda: Prosobranchia). Malacological Review, Ann Arbor, 26: 9-14.

Kleewein, D. 1999. Population size, density, spatial distribution and dispersal in an Austrian population of the land snail Arianta arbustorum styriaca (Gastropoda: Helicidae). Journal of Molluscan Studies, Londres, 65: 303-315.

Ковак, J. 2001. Light, gravity and cospecifics as cues to site selection and attachment behaviour of juvenile and adult Dreissena polymorpha Pallas, 1971. Journal of Molluscan Studies, Londres, 67: 183-189.

Martell, K.A.; V. Tunnicliffe \& I.R. MacDonald. 2002. Biological features of a buccinid whelk (Gastropoda, Neogastropoda) at the endeavor venefields of Juan de Fuça ridge, northeast Pacific. Journal of Molluscan Studies, Londres, 68: 45-53.

Morton, B.; G. Blackmore \& C.T. KwoK. 2002. Corallivory and prey choice by Drupella rugosa (Gastropoda: Muricidae) in Hong Kong. Journal of Molluscan Studies, Londres, 68: 217-223.

ReIse, H. 1995. Mating behaviour of Derosceras rodnae Grosu \& Lupu, 1965 and D. praecox Wiktor, 1966 (Pulmonata: Agriolimacidae). Journal of Molluscan Studies, Londres, 61: 35-330. 
Skingsley, D.R.; A.J. White \& A. Weston. 2000. Analysis of pulmonate mucus by infrared spectroscopy. Journal of Molluscan Studies, Londres, 66: 363-371.

Stephenson, J.W. 1979. The functioning of the sense organs associated with feeding behaviour in Deroceras reticulatum (Müll.). Journal of Molluscan Studies, Londres, 45: 167171.

Recebido em 09.VI.2005; aceito em 07.IV.2006.
Towsend, C.R. 1974. Mucus trail following by the snail Biomphalaria glabrata (Say). Animal Behaviour, Nedlands, 22: 170-177.

Vianey-Liaud, M. \& G. Dussart. 2002. Aspects of pairing and reproduction in the hermaphrodite freshwater snail Biomphalaria glabrata (Gastropoda; Pulmonata). Journal of Molluscan Studies, Londres, 68: 243-248. 